\title{
Histologic findings after explantation of a modified expanded polytetrafluoroethylene graft used for clinical systemic-to-pulmonary shunting
}

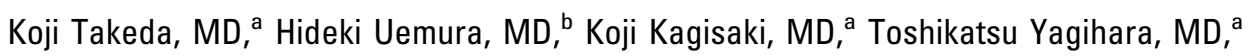

and Paul Silvagni, DVM, PhD, ${ }^{\mathrm{C}}$ Osaka, Japan, London, United Kingdom, and Flagstaff, Ariz

A fter construction of a systemic-to-pulmonary shunt with an expanded polytetrafluoroethylene (ePTFE) graft, a perigraft seroma may develop as a result of seepage of serious fluid through the prosthesis. ${ }^{1}$ In an effort to prevent this complication, a modified ePTFE pediatric shunt graft covered with a low-permeability reinforcing film (pore size $<1 \mu \mathrm{m}$ ) has been developed (Gore-Tex Vascular Graft Configured for Pediatric Shunt; W. L. Gore \& Associates, Inc, Flagstaff, Ariz). We describe our initial clinical experience with this graft, focusing on histologic studies performed after explantation.

\section{Patients and Methods}

Beginning in 2001, the modified graft was implanted in 13 consecutive patients (aged 13 days to 6 years) for construction of a modified Blalock-Taussig shunt $(n=9)$ or a central shunt from the ascending aorta to a standard $18-\mathrm{mm}$ ePTFE graft $(n=4)$. The shunt graft was routinely covered with an ePTFE surgical membrane to avoid adhesions and facilitate its subsequent dissection. All patients received continuous intravenous infusions of heparin immediately after surgery. When oral intake resumed, warfarin and an antiplatelet agent were administered.

Forty-nine to 653 days after implantation, 14 shunt graft specimens ( 2 from 1 patient) were obtained for histologic analysis at takedown of the shunt in preparation for additional surgery. In patients with a central shunt, small pieces of the 18-mm standard ePTFE graft were removed to tailor it for the inferior caval venous channel, and these specimens were also analyzed. The histologic studies included hematoxylin and eosin staining, trichrome staining, and immunohistochemical assessments for factor VIII (a marker for endothelium) and smooth muscle actin antigen.

\footnotetext{
From the Department of Cardiovascular Surgery, National Cardiovascular

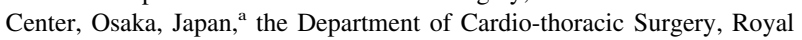
Brompton Hospital, London, United Kingdom, ${ }^{b}$ and W. L. Gore \& Associates, Inc, Flagstaff, Ariz.

Received for publication March 7, 2005; accepted for publication March 22, 2005.

Address for reprints: Toshikatsu Yagihara, MD, Department of Cardiovascular Surgery, National Cardiovascular Center, 5-7-1 Fujishirodai, Suita, Osaka 565-8565, Japan (E-mail: yagihara@hsp.ncvc.go.jp).

J Thorac Cardiovasc Surg 2005;130:934-5

$0022-5223 / \$ 30.00$

Copyright $\odot 2005$ by The American Association for Thoracic Surgery doi:10.1016/j.jtcvs.2005.03.034
}

\section{Results}

Clinical examinations showed that all shunt grafts remained patent during use. No seromas were observed on echocardiography, on chest roentgenography, or during inspection of the graft specimens
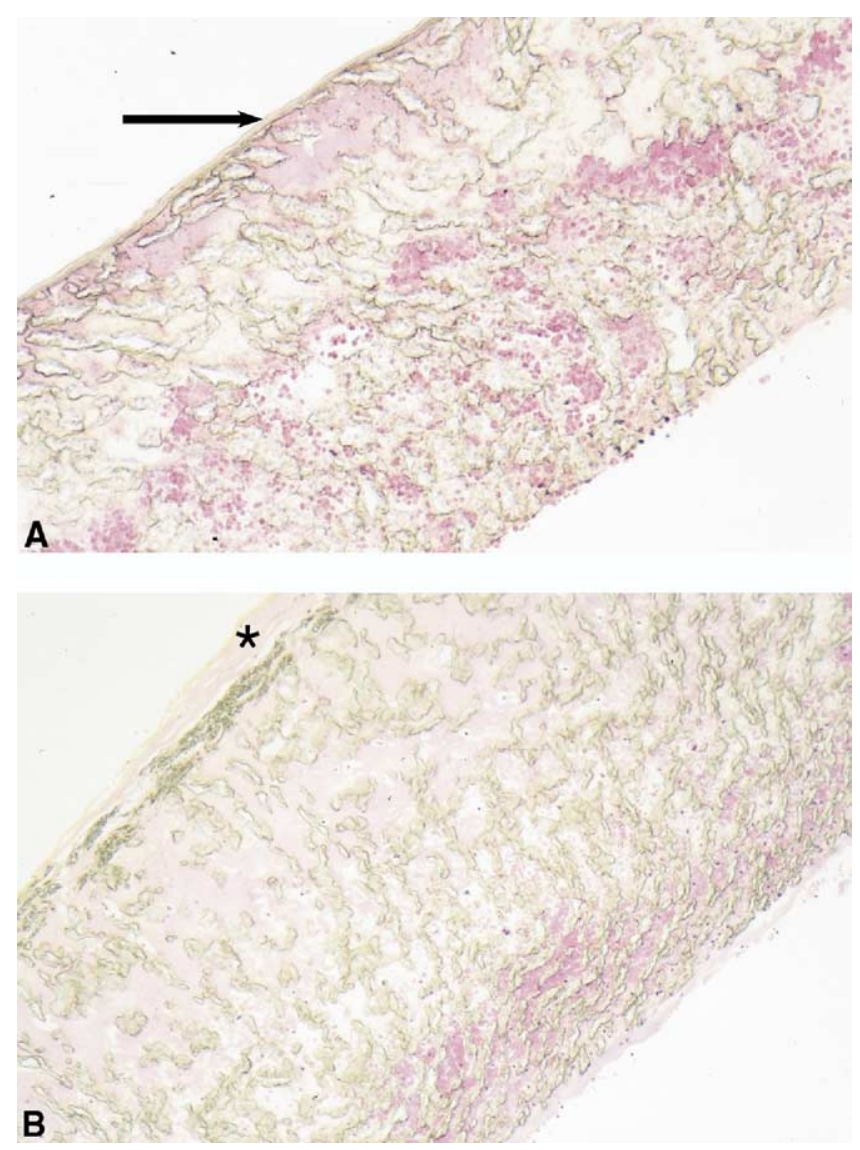

Figure 1. Histologic studies of explanted ePTFE grafts. A, Specimen from modified graft used for central shunt, obtained about 201 days after implantation. Proteinaceous fluids are present within graft interstices (pink) but not outside outer membrane (upper right). Thin, low-permeability film is visible at ablumen (arrow). There is no neointimal proliferation along lumen (lower left; hematoxylin-eosin stain, original magnification $\times 50)$. B, Specimen from standard 18-mm ePTFE graft, obtained about 210 days after implantation. Small accumulation of proteinaceous fluid (asterisk) appears on abluminal surface of graft (hematoxylin-eosin stain, original magnification $\times 25$ ). 


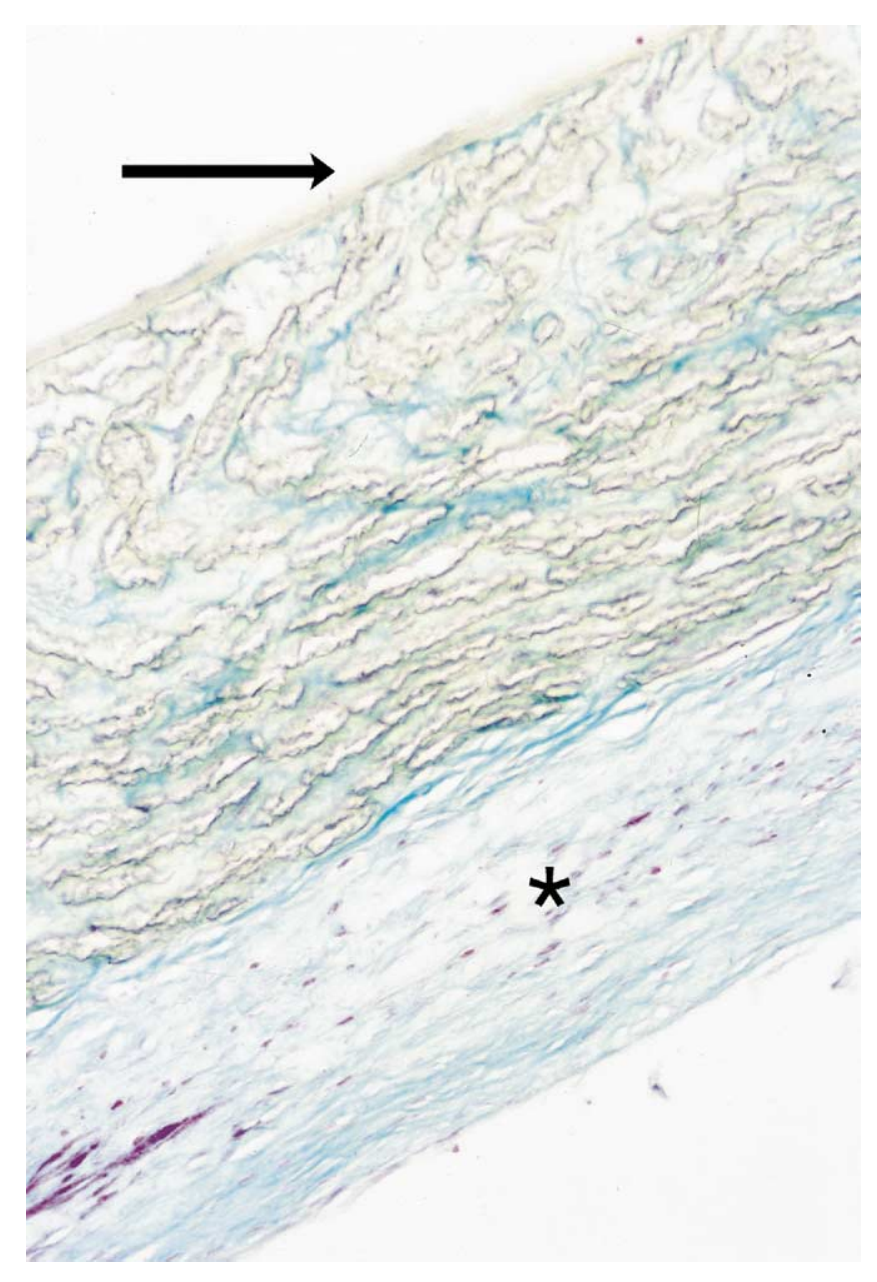

Figure 2. Specimen from explanted modified graft used for Blalock-Taussig shunt. Collagenous neointima (asterisk) lines lumen, and collagen fibers penetrate luminal surface. Low-permeability membrane (arrow) is visible at ablumen (trichrome stain, original magnification $\times 50$ ).

at explantation. In the 1 patient who required a second thoracotomy to treat persistent chylothorax, no evidence of serous leakage was observed around the implanted graft at operation.

At harvesting, all shunt graft specimens were macroscopically patent, and there were no perigraft accumulations of fibrinous tissue. Microscopy showed that proteinaceous fluid had infiltrated the interstices of the grafts but had not leaked through the outer film layer (Figure 1, A). In contrast, 3 of the 4 standard ePTFE graft specimens showed leakage of fluid through the outer graft wall (Figure 1, B). In 13 of the 14 shunt graft specimens, the outer graft surface had no tissue attachment; in the other, the graft was encapsulated by mature collagenous (trichrome-positive) tissue (Figure 2). No specimen had evidence of mineralization, diffuse inflammation, or infection.
No thrombotic tissue was present in the lumen of any of the shunt grafts, but all had a neointima. The Pearson correlation coefficient demonstrated no correlation between maximum neointimal thickness and length of time the graft was implanted $(r=$ 0.15 ). The neointima was composed of circumferentially arranged spindle cells within a laminar collagen stroma. The lumina were lined by flattened epithelial cells positive for factor VIII antigen and consistent with endothelium. Spindle cells containing smooth muscle actin were located in both the collagenous neointima and the interstices of the shunt grafts. Collagen fibers had penetrated the luminal interstices of the grafts.

\section{Discussion}

Many factors have been postulated to contribute to perigraft seroma formation, including use of heparin, ${ }^{2}$ inappropriate graft handling, ${ }^{3}$ shunt size, and the presence of an inhibitor that prevents maturation or proliferation of perigraft fibroblasts. ${ }^{4}$ In our experience with 150 standard ePTFE pediatric shunts, we observed important seromas only after complicated procedures such as pulmonary arterial reconstruction with a heterologous pericardial roll. ${ }^{5} \mathrm{We}$ therefore assume that persistent high stress on the wall of a shunt graft is one of the mechanisms of seroma formation.

The histologic findings in this study suggest that the exterior reinforcing film on the modified ePTFE shunt graft increases resistance to leakage of proteinaceous fluid through the graft wall. In contrast, fluid leakage was observed in the explanted standard ePTFE graft specimens. The data indicate that use of the modified shunt graft may prevent seroma formation after pediatric shunt procedures.

A concern pertaining to use of nonporous grafts is that excessive intimal proliferation may eventually result in occlusion of the lumen by the neointima, especially in small-diameter grafts such as those used for pediatric shunts. There were no occlusions in the shunt grafts in our patients, but we cannot rule out the possibility that occlusion might eventually develop. On the other hand, we found no correlation between maximum neointimal thickness in the shunt grafts and duration of implantation. Patients with modified shunt graft implants should be monitored closely.

\section{References}

1. Noyez L, Daenen W. The modified polytetrafluoroethylene BlalockTaussig shunt. Case report of an unusual complication. J Thorac Cardiovasc Surg. 1987;94:634-5.

2. Berger RM, Bol-Raap G, Hop WJ, Bogers AJ, Hess J. Heparin as a risk factor for perigraft seroma complicating the modified Blalock-Taussig shunt. J Thorac Cardiovasc Surg. 1998;116:286-93.

3. Tabata R, Kobayahshi T, Mori A, Matsuno S, Watarida S, Onoe M, et al. A computer simulation of the plasma leakage through a vascular prosthesis made of expanded polytetrafluoroethylene. J Thorac Cardiovasc Surg. 1993;105:598-604.

4. Ahn SS, Williams DE, Thye DA, Cheng KQ, Lee DA. The isolation of a fibroblast growth inhibitor associated with perigraft seroma. $J$ Vasc Surg. 1994;20:202-8.

5. Uemura H, Yagihara T, Kawashima Y, Yamashita K, Kamiya T. Intrapulmonary reconstruction of pulmonary arteries using a heterologous pericardial roll. Ann Thorac Surg. 1995;59:1464-70. 